\title{
Phase-Field Simulation of the Effect of Elastic Inhomogeneity on Microstructure Evolution in Ni-Based Superalloys
}

\author{
Yuhki Tsukada $^{1, *}$, Yoshinori Murata ${ }^{1}$, Toshiyuki Koyama ${ }^{2}$ and Masahiko Morinaga ${ }^{1}$ \\ ${ }^{1}$ Department of Materials, Physics and Energy Engineering, Graduate School of Engineering, \\ Nagoya University, Nagoya 464-8603, Japan \\ ${ }^{2}$ National Institute for Materials Science, Tsukuba 305-0047, Japan
}

Phase-field simulation is performed to examine the effect of elastic inhomogeneity between the $\gamma$ and $\gamma^{\prime}$ phases on microstructure evolution of the $\gamma^{\prime}$ phase in Ni-based superalloys. For solving the elastic equilibrium equation numerically in the elastically inhomogeneous system, an iterative approach is used. In this simulation, both elastic anisotropy and shear modulus are varied independently based on the elastic constants of a practical Ni-based superalloy. Four different types of anti-phase domains in the $\gamma^{\prime}$ phase are also considered in this simulation. The variation of elastic anisotropy affected significantly the manner of both morphology of the $\gamma^{\prime}$ phase and distribution function of the $\gamma^{\prime}$ particle size, whereas the variation of shear modulus did not affect them. [doi:10.2320/matertrans.MBW200826]

(Received October 20, 2008; Accepted February 10, 2009; Published March 25, 2009)

Keywords: phase-field model, nickel-based alloys, elastic inhomogeneity

\section{Introduction}

Ni-based superalloys consisting of the $\gamma^{\prime}$ phase $\left(\mathrm{L}_{2}\right.$ structure) precipitated in the $\gamma$ phase with face-centered cubic lattice are applied to gas turbine materials because of their excellent mechanical properties such as creep strength at high temperatures. It is necessary to control the $\gamma^{\prime}$ volume fraction, morphology and size distribution of the $\left(\gamma+\gamma^{\prime}\right)$ two-phase microstructure, since the mechanical properties of the superalloys are strongly related to them.

In recent years, the phase-field model has been applied for simulating microstructure evolution in Ni-Al alloys. ${ }^{1-4)}$ The phase-field model describes a microstructure using a set of conserved or non-conserved field variables. The microstructure evolution can be simulated by a set of equations governing the evolution of the fields under the condition of achieving the minimum of the total free energy of the system. In previous works on Ni-based alloys, ${ }^{1,2,4)}$ the difference in elastic constants between the $\gamma$ and $\gamma^{\prime}$ phases (elastic inhomogeneity) have not been considered in the calculation of the elastic strain energy, because the elastic inhomogeneity is less than $7 \%$ in the wide range of temperatures in $\mathrm{Ni}$ $\mathrm{Al}$ binary system. ${ }^{5)}$ However, it is important to understand the effect of the elastic inhomogeneity on the microstructure evolution, because the elastic inhomogeneity has become about $13 \%$ in a practical Ni-based superalloy at high temperatures. ${ }^{6)}$

There are some models considering the elastic inhomogeneity in the phase-field model. ${ }^{7,8)}$ For example, $\mathrm{Hu}$ and $\mathrm{Chen}^{8)}$ proposed a very efficient phase-field model for elastically inhomogeneous systems, in which an iterative approach is used for solving the elastic equilibrium equation numerically. They concluded that it required a rather strong elastic inhomogeneity $(>50 \%)$ to produce precipitate morphology different dramatically from one obtained in elastically homogeneous systems. However, the effect of the elastic inhomogeneity in Ni-based alloys should be assessed in the condition taking into account the four types of anti-

*Graduate Student, Nagoya University phase domains existing in the $\gamma^{\prime}$ phase, because Wang et al. ${ }^{1)}$ reported that the ordered nature of the precipitate played an important role in the microstructure evolution.

The purpose of this study is to examine the effect of the elastic inhomogeneity on microstructure evolution of the $\gamma^{\prime}$ phase in Ni-based superalloys by the phase-field simulation. In the elastic energy calculation, the elastic constants are based on a practical alloy and the iterative approach is used for solving the local displacement vector. Four different types of anti-phase domains in the $\gamma^{\prime}$ phase are also considered in this simulation.

\section{Calculation Model}

In order to simulate microstructure change of the $\gamma$ and $\gamma^{\prime}$ phases, the volume fraction of the $\gamma^{\prime}$ phase $f(\mathbf{r}, t)$ and four artificial order parameters $\phi_{i}(\mathbf{r}, t)(i=1,2,3,4)$, which describe the four different domains in the $\mathrm{L}_{2}$ phase structure, are chosen as field variables. These field variables vary spatially (r) and temporally $(t)$. Usually, alloy composition $c(\mathbf{r}, t)$ is used as a field variable, ${ }^{1-4)}$ but $f(\mathbf{r}, t)$ is used instead of $c(\mathbf{r}, t)$ in this study, because $f(\mathbf{r}, t)$ is suitable to treat the multi-component systems when the phase-field model is applied to the practical Ni-based alloys. The temporal evolution of the field variables is given by solving the following Cahn-Hilliard and Ginzburg-Landau equations:

$$
\begin{aligned}
& \frac{\partial f(\mathbf{r}, t)}{\partial t}=M \nabla^{2} \frac{\delta G_{\mathrm{sys}}}{\delta f(\mathbf{r}, t)}, \\
& \frac{\partial \phi_{i}(\mathbf{r}, t)}{\partial t}=-L \frac{\delta G_{\mathrm{sys}}}{\delta \phi_{i}(\mathbf{r}, t)} \quad(i=1,2,3,4),
\end{aligned}
$$

where $G_{\text {sys }}$ is the total free energy of the system, $M$ is the diffusion mobility and $L$ is the structural relaxation coefficient. The total free energy of the system is given by,

$$
G_{\mathrm{sys}}=\int_{\mathbf{r}}\left[G_{\text {chem }}\left(f, \phi_{i}\right)+E_{\text {grad }}\left(\phi_{i}\right)+E_{\text {str }}\left(\phi_{i}\right)\right] d \mathbf{r},
$$

where $G_{\text {chem }}$ is the chemical free energy density, $E_{\text {grad }}$ is the gradient energy density and $E_{\text {str }}$ is the elastic strain energy density. The chemical free energy density is expressed as, 


$$
\begin{aligned}
G_{\text {chem }}= & \left\{1-h\left(\phi_{i}\right)\right\} G_{\text {chem }}^{\mathrm{m}}\left(f_{\mathrm{m}}\right) \\
& +h\left(\phi_{i}\right) G_{\text {chem }}^{\mathrm{p}}\left(f_{\mathrm{p}}\right)+w g\left(\phi_{i}\right),
\end{aligned}
$$

where $w$ is the double-well potential height. ${ }^{3,4)}$ In this study, the chemical free energy density of the $\gamma$ matrix and the $\gamma^{\prime}$ precipitate, $G_{\mathrm{chem}}^{\mathrm{m}}$ and $G_{\mathrm{chem}}^{\mathrm{p}}$, are given as,

$$
\begin{aligned}
G_{\text {chem }}^{\mathrm{m}} & =\frac{1}{2} W_{1} f^{2}, \\
G_{\text {chem }}^{\mathrm{p}} & =\frac{1}{2} W_{2}(1-f)^{2},
\end{aligned}
$$

where $W_{1}$ and $W_{2}$ are the coefficients determined by the Gibbs energy calculation based on the sub-lattice model using the thermodynamic database. The functions $h\left(\phi_{i}\right)$ and $g\left(\phi_{i}\right)$ are selected as,

$$
\begin{aligned}
& h\left(\phi_{i}\right)=\sum_{i=1}^{4}\left[\phi_{i}^{3}\left(10-15 \phi_{i}+6 \phi_{i}^{2}\right)\right], \\
& g\left(\phi_{i}\right)=\sum_{i=1}^{4}\left[\phi_{i}^{2}\left(1-\phi_{i}\right)^{2}\right]+\alpha \sum_{i=1}^{4} \sum_{j \neq i}^{4} \phi_{i}^{2} \phi_{j}^{2} .
\end{aligned}
$$

Employing the description proposed by Kim et al. ${ }^{9}{ }^{9}$ the interface region is regarded as a mixture of the $\gamma$ and $\gamma^{\prime}$ phases with different volume fractions of the $\gamma^{\prime}$ phase but with equal chemical potentials:

$$
\begin{aligned}
& f=\left\{1-h\left(\phi_{i}\right)\right\} f_{\mathrm{m}}+h\left(\phi_{i}\right) f_{\mathrm{p}} \\
& \left(\frac{\partial G_{\text {chem }}^{\mathrm{m}}}{\partial f}\right)_{f=f_{\mathrm{m}}}=\left(\frac{\partial G_{\text {chem }}^{\mathrm{p}}}{\partial f}\right)_{f=f_{\mathrm{p}}} .
\end{aligned}
$$

The gradient energy density is estimated from the artificial order parameters as,

$$
E_{\text {grad }}=\frac{1}{2} \kappa_{\phi} \sum_{i=1}^{4}\left(\nabla \phi_{i}\right)^{2},
$$

where $\kappa_{\phi}$ is the gradient energy coefficient of the order parameter. ${ }^{10)}$ The elastic strain energy density arising from the lattice misfit between the $\gamma$ and $\gamma^{\prime}$ phases is estimated based on the micromechanics: ${ }^{11,12}$

$$
E_{\mathrm{str}}=\frac{1}{2} C_{i j k l} \varepsilon_{i j}^{e l}(\mathbf{r}, t) \varepsilon_{k l}^{e l}(\mathbf{r}, t) .
$$

The elastic strain, $\varepsilon_{k l}^{e l}$, is expressed as,

$$
\varepsilon_{k l}^{e l}(\mathbf{r}, t)=\varepsilon_{k l}^{c}(\mathbf{r}, t)-\varepsilon_{k l}^{0}(\mathbf{r}, t),
$$

where $\varepsilon_{k l}^{c}$ and $\varepsilon_{k l}^{0}$ represent the constrained strain and eigenstrain, respectively. The eigenstrain is expressed as,

$$
\varepsilon_{k l}^{0}(\mathbf{r}, t)=\varepsilon_{0} \delta_{k l}\left\{h\left(\phi_{i}\right)-\overline{h\left(\phi_{i}\right)}\right\},
$$

and

$$
\varepsilon_{0}=\frac{a_{\mathrm{m}}-a_{\mathrm{p}}}{a_{\mathrm{m}}},
$$

where $\varepsilon_{0}$ represents the lattice misfit and $\delta_{k l}$ is the Kronecker delta function. In eq. (14), $a_{\mathrm{m}}$ and $a_{\mathrm{p}}$ are the lattice parameters of the $\gamma$ matrix and the $\gamma^{\prime}$ precipitate, respectively. In eq. (12), the constrained strain can be expressed as,

$$
\varepsilon_{k l}^{c}(\mathbf{r})=\frac{1}{2}\left\{\frac{\partial u_{k}(\mathbf{r})}{\partial r_{l}}+\frac{\partial u_{l}(\mathbf{r})}{\partial r_{k}}\right\},
$$

where $\mathbf{u}$ represents the local displacement vector.
The Hooke's law gives the local elastic stress as $\sigma_{i j}^{e l}(\mathbf{r})=C_{i j k l} \varepsilon_{k l}^{e l}(\mathbf{r})$, and the local elastic constants $C_{i j k l}$ is assumed to be $C_{i j k l}=\left\{1-h\left(\phi_{i}\right)\right\} C_{i j k l}^{\mathrm{m}}+h\left(\phi_{i}\right) C_{i j k l}^{\mathrm{p}}$ in eq. (11), where $C_{i j k l}^{\mathrm{m}}$ and $C_{i j k l}^{\mathrm{p}}$ represent the elastic constants of the $\gamma$ matrix and the $\gamma^{\prime}$ precipitate, respectively. From the local mechanical equilibrium equation, i.e., $\partial \sigma_{i j}^{e l} / \partial r_{j}=0$, the local displacement vector in eq. (15) is solved in Fourier space by applying the iterative approach. ${ }^{8)}$ The number of iterations depends on the degree of the elastic inhomogeneity.

\section{Results}

Phase-field simulation at a temperature of $1373 \mathrm{~K}$ was performed by solving the two sets of eqs. (1) and (2) numerically, using the explicit method under the periodic boundary conditions. The coefficients of the chemical free energy density in eqs. (5) and (6) were determined as $W_{1}=$ $1.20 \times 10^{3}$ and $W_{2}=1.45 \times 10^{3} \mathrm{~J} \cdot \mathrm{mol}^{-1}$ based on the Gibbs energy calculation using the thermodynamic database. ${ }^{13)}$ By fitting to the interfacial energy density, which has the value of $14.2 \mathrm{~mJ} \cdot \mathrm{m}^{-2}$ in Ni-Al binary alloys, ${ }^{14)}$ the double-well potential height and the gradient energy coefficient were determined as $w=1.7 \times 10^{7} \mathrm{~J} \cdot \mathrm{m}^{-3}$ and $\kappa_{\phi}=$ $2.1 \times 10^{-10} \mathrm{~J} \cdot \mathrm{m}^{-1}$, respectively. Elastic constants of the TMS-26 alloy at $1373 \mathrm{~K}\left(C_{11}^{\mathrm{m}}=184, C_{12}^{\mathrm{m}}=135, C_{44}^{\mathrm{m}}=\right.$ $\left.\left.88.1, C_{11}^{\mathrm{p}}=209, C_{12}^{\mathrm{p}}=142, C_{44}^{\mathrm{p}}=90.4 \mathrm{GPa}\right)^{6}\right)$ were employed in this simulation, and the volume fraction of the $\gamma^{\prime}$ phase in the equilibrium state was set to be $55 \%$. $^{6}$ ) The lattice misfit was set to be $\varepsilon_{0}=-0.003$, which is close to the value for TMS-26 alloy. ${ }^{6)}$ Except for the elastic constants, physical constants for $\mathrm{Ni}-\mathrm{Al}$ binary system were employed in this study, because the focus of this study is to examine the effect of the elastic inhomogeneity on the microstructure evolution qualitatively. The simulation was started from the supersaturated solid solution with a random noise of the field variables $\left(f, \phi_{i}\right)$.

\subsection{Morphology}

Figure 1 shows 2D morphological evolution of the $\gamma^{\prime}$ phase in the late stage of coarsening at $1373 \mathrm{~K}$ calculated from the phase-field simulation using the elastic constants of the TMS-26 alloy. The $\gamma^{\prime}$ phase is expressed as white area in Fig. 1. The cuboidal shape of the $\gamma^{\prime}$ phase arranged along the $\langle 10\rangle$ crystallographic direction of the $\gamma$ matrix is observed due to the anisotropic elastic interaction. Furthermore, the coalescence of the neighboring $\gamma^{\prime}$ phases with the same order parameter is observed as marked by arrows in Figs. 1(c) and (d), the coalescence of which has been also reported from the simulation in $\mathrm{Ni}-\mathrm{Al}$ alloys. ${ }^{4)}$

Three independent elastic quantities exist in cubic system, i.e., the bulk modulus $\left(B=C_{11}+C_{12}\right)$, the shear modulus $\left(G=2 C_{44}\right)$ and the anisotropy $\left(\delta=2 C_{44} /\left(C_{11}-C_{12}\right)\right)$. To examine the effect of elastic inhomogeneity, we changed only the anisotropy or the shear modulus while keeping the other two quantities in the simulation. On the basis of the elastic constants of the TMS-26 alloy, each quantity was changed under the limitation that the Young's modulus of the $\gamma^{\prime}$ phase is larger than that of the $\gamma$ phase along all the crystallographic directions $\left(E^{\mathrm{m}}<E^{\mathrm{p}}\right)$. Each of the quantities for the elastic constants of the TMS-26 alloy are 

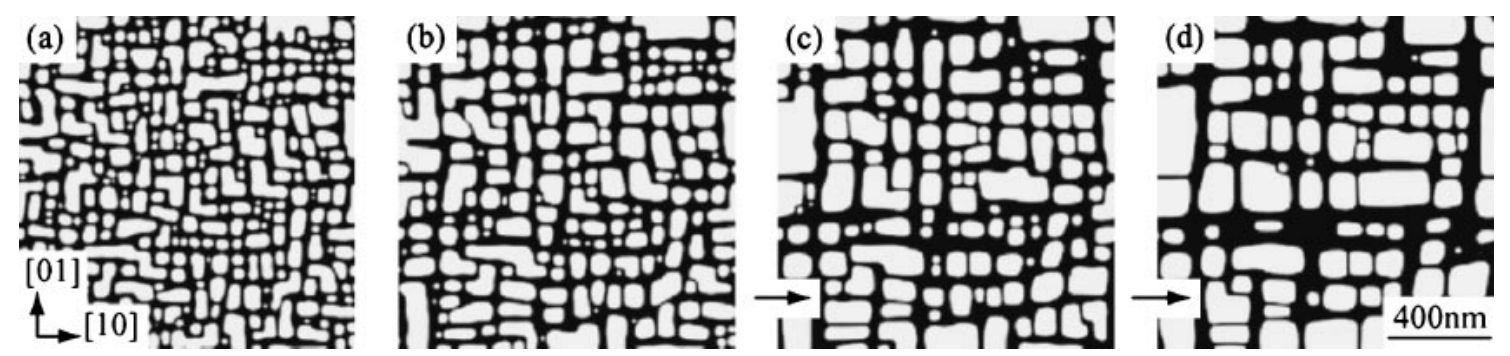

Fig. 1 Morphological evolution of the $\gamma^{\prime}$ phase calculated from 2D phase-field simulation at $1373 \mathrm{~K}$ using the elastic constants of a practical Ni-based superalloy. (a) $t=10000$, (b) $t=20000$, (c) $t=40000$ and (d) $t=80000$. All time expressed here are dimensionless time steps.
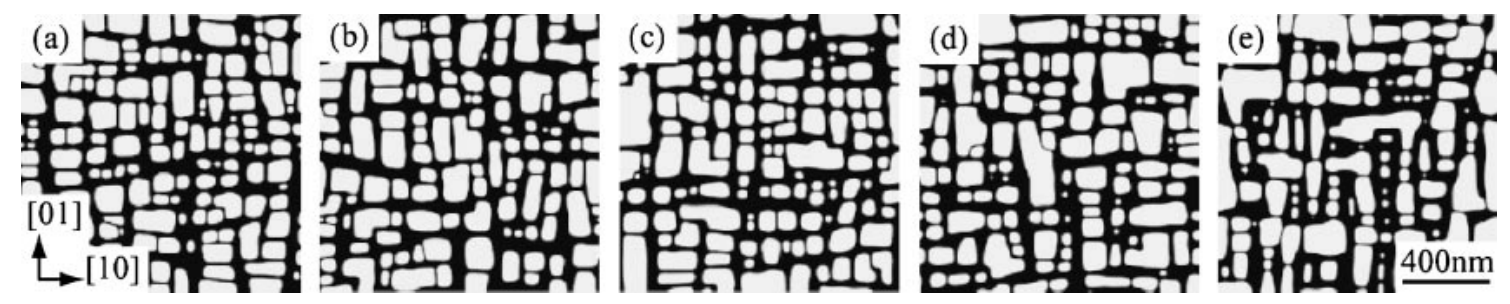

Fig. 2 Dependence of the $\gamma^{\prime}$ phase morphology on elastic anisotropy in the $\gamma$ matrix and $\gamma^{\prime}$ precipitate. $\left(\delta^{\mathrm{m}}, \delta^{\mathrm{p}}\right)$ : (a) $(6.07,2.17),(\mathrm{b})(4.51$, $2.40)$, (c) $(3.59,2.69)$, (d) $(2.98,3.07)$ and (e) $(2.55,3.57) . \delta$ is $2 C_{44} /\left(C_{11}-C_{12}\right)$
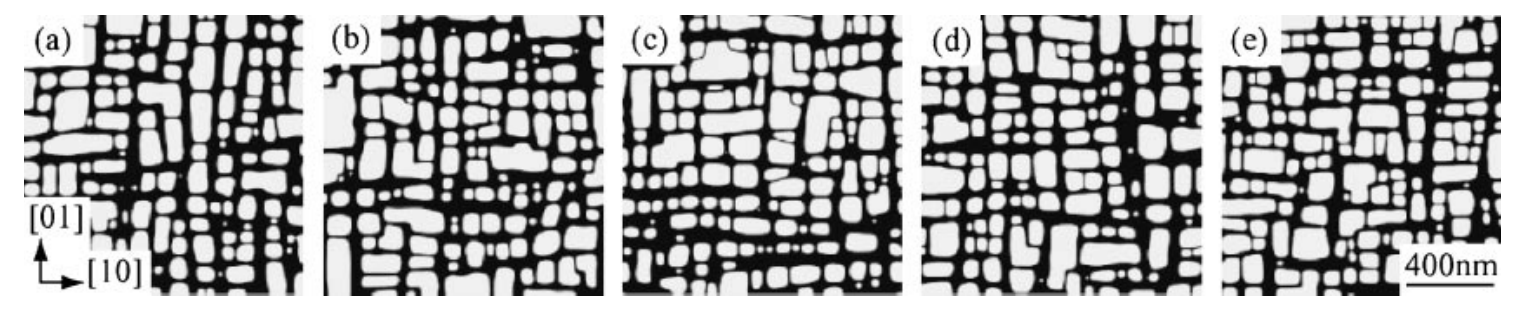

Fig. 3 Dependence of the $\gamma^{\prime}$ phase morphology on shear modulus in the $\gamma$ matrix and $\gamma^{\prime}$ precipitate. (a) $\kappa=0.90$, (b) $\kappa=1.02$, (c) $\kappa=1.16$, (d) $\kappa=1.32$ and (e) $\kappa=1.74 . \kappa$ is $C_{44}^{\mathrm{p}} / C_{44}^{\mathrm{m}}$.

$\left(B^{\mathrm{m}}, B^{\mathrm{p}}\right)=(319,351),\left(G^{\mathrm{m}}, G^{\mathrm{p}}\right)=(176,180)$ and $\left(\delta^{\mathrm{m}}, \delta^{\mathrm{p}}\right)=$ $(3.59,2.69)$. Here, the superscripts $m$ and $\mathrm{p}$ represent the elastic constants of the $\gamma$ and $\gamma^{\prime}$ phases, respectively.

Figure 2 shows the results of the phase-field simulation obtained in the late stage of coarsening of the $\gamma^{\prime}$ phase, indicating the dependence of the $\gamma^{\prime}$ morphology on the anisotropy in the $\gamma$ and $\gamma^{\prime}$ phases. The anisotropy is varied in five sets as $\left(\delta^{\mathrm{m}}, \delta^{\mathrm{p}}\right)=(6.07,2.17),(4.51,2.40),(3.59,2.69)$, $(2.98,3.07),(2.55,3.57)$. In this simulation, both bulk and shear modulus are kept to be constant. It is observed in Fig. 2 that the cuboidal $\gamma^{\prime}$ phase shows irregularity when the ratio of $\delta^{\mathrm{p}}$ to $\delta^{\mathrm{m}}$ becomes large (see Fig. 2(e)). Figure 3 shows the simulation results obtained in the late stage of coarsening of the $\gamma^{\prime}$ phase, indicating the dependence of the $\gamma^{\prime}$ morphology on the shear modulus in the $\gamma$ and $\gamma^{\prime}$ phases. The shear modulus is varied in five sets as $\left(\kappa=C_{44}^{\mathrm{p}} / C_{44}^{\mathrm{m}}=0.90,1.02\right.$, $1.16,1.32,1.74)$. In this simulation, both bulk modulus and anisotropy are kept to be constant. It is found that the elastic inhomogeneity caused by the different ratios of the shear modulus in the $\gamma$ and $\gamma^{\prime}$ phases affects scarcely the morphology of the $\gamma^{\prime}$ phase.

\subsection{Particle size distribution}

From the simulation results, scaled particle size distribution of the $\gamma^{\prime}$ phase was obtained by measuring the fraction of the $\gamma^{\prime}$ particles having a radius $(r)$ within a size interval which was normalized by the average radius $(\bar{r})$. The particle size distribution is obtained from the result for each time step. In this study, according to a similar way to Vaithyanathan and Chen, ${ }^{2)}$ averaged particle size distribution was constructed from the results for four different time steps corresponding to Fig. 1. Figure 4 shows the scaled particle size distributions obtained from simulation results for five different conditions corresponding to Fig. 2, in which the anisotropy in the $\gamma$ and $\gamma^{\prime}$ phases are changed independently. It is shown that the spectrum becomes broad with increasing the ratio of $\delta^{\mathrm{p}}$ to $\delta^{\mathrm{m}}$, while the radius showing the maximum size distribution (hereafter called as peak radius) does not change and it is almost at 0.75 in the normalized radius. Figure 5 shows the scaled particle size distributions obtained from simulation results for five different conditions corresponding to Fig. 3, in which only the ratio of the shear modulus in the $\gamma$ and $\gamma^{\prime}$ phases is changed independently. From Fig. 5, it is found that both the peak radius and the spectrum shape are not affected by the variation of the shear modulus.

The effect of the elastic inhomogeneity on the standard deviation of the particle size distribution is summarized in Table 1. It is obvious that the variation of the anisotropy broadens the spectrum of particle size distribution but that the variation of the shear modulus does not affect it. 

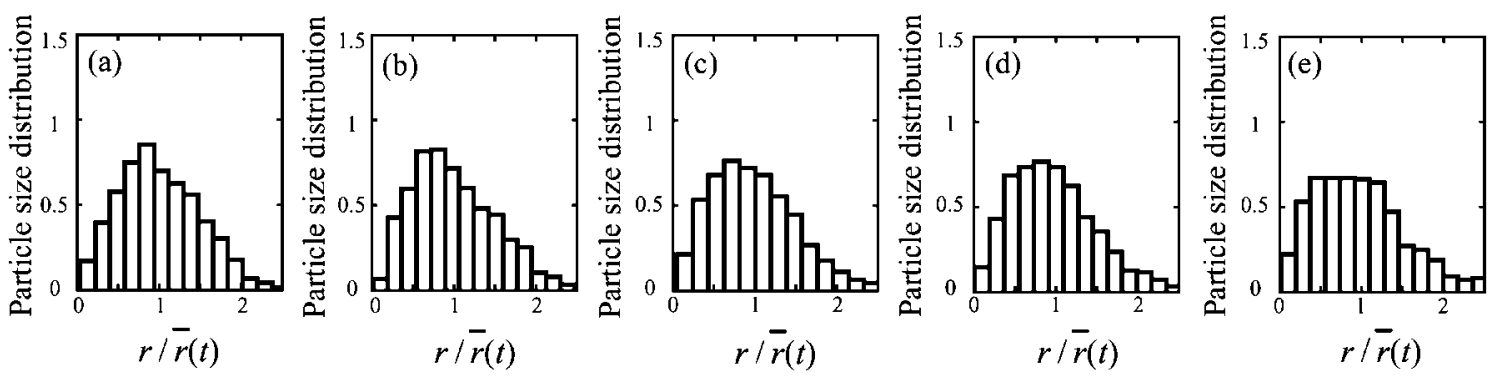

Fig. 4 Scaled particle size distributions obtained from simulation results for five different conditions corresponding to Fig. 2, varying only elastic anisotropy in the $\gamma$ matrix and $\gamma^{\prime}$ precipitate. $\left(\delta^{\mathrm{m}}, \delta^{\mathrm{p}}\right)$ : (a) $(6.07,2.17),(\mathrm{b})(4.51,2.40),(\mathrm{c})(3.59,2.69),(\mathrm{d})(2.98,3.07)$ and (e) $(2.55,3.57)$. In all cases, averaged results are shown which are obtained from simulation results for four different time steps corresponding to Fig. 1.
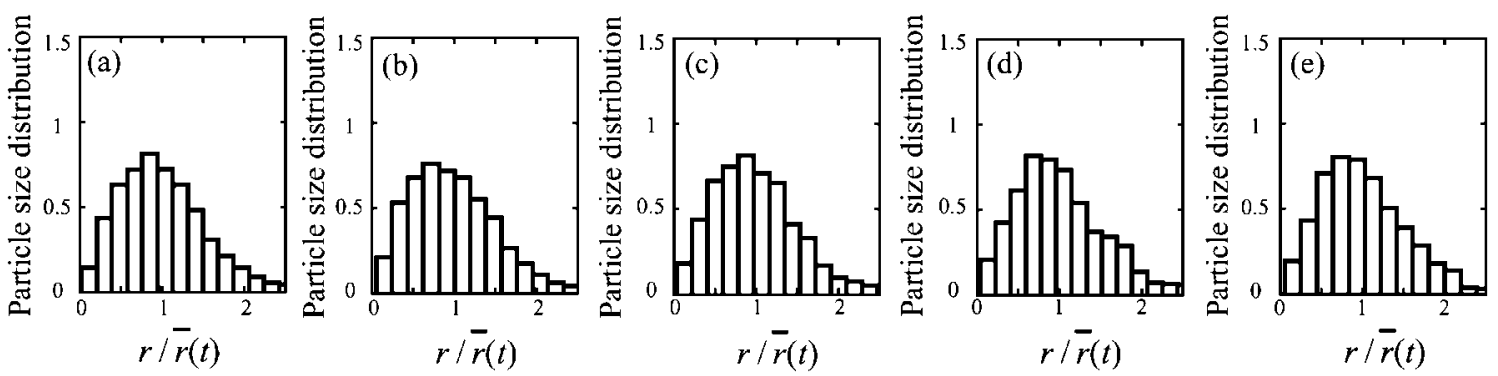

Fig. 5 Scaled particle size distributions obtained from simulation results for five different conditions corresponding to Fig. 3, varying only shear modulus in the $\gamma$ matrix and $\gamma^{\prime}$ precipitate. (a) $\kappa=0.90$, (b) $\kappa=1.02$, (c) $\kappa=1.16$, (d) $\kappa=1.32$ and (e) $\kappa=1.74$. In all cases, averaged results are shown which are obtained from simulation results for four different time steps corresponding to Fig. 1.

Table 1 Standard deviation of size distribution curves of the $\gamma^{\prime}$ particles obtained from different simulation results as shown in Figs. 4 and 5.

\begin{tabular}{ccc}
\hline \multicolumn{2}{c}{ Simulation condition } & Standard deviation \\
\hline \multirow{3}{*}{$(6.07,2.17)$} & 0.478 \\
$(4.51,2.40)$ & 0.504 \\
$(3.59,2.69)$ & 0.535 \\
& $(2.98,3.07)$ & 0.572 \\
& $(2.55,3.57)$ & 0.593 \\
\hline$\kappa$ & 0.90 & 0.521 \\
& 1.02 & 0.535 \\
& 1.16 & 0.513 \\
& 1.32 & 0.523 \\
& 1.74 & 0.496 \\
\hline
\end{tabular}

\section{Discussion}

From the phase-field simulation based on the variation of only the anisotropy in the $\gamma$ and $\gamma^{\prime}$ phases, it is found that the $\gamma^{\prime}$ phase shows unstable shape as shown in Fig. 2(e) when the ratio of $\delta^{p}$ to $\delta^{\mathrm{m}}$ becomes large. In the previous work, $\mathrm{Hu}$ and $\mathrm{Chen}^{8)}$ showed that elastic energy became low when hard precipitates were surrounded by soft matrix comparing to that soft precipitates were surrounded by hard matrix. The simulation condition of Fig. 2(e) is the case that the Young's modulus of the $\gamma^{\prime}$ phase is just slightly larger than that of the $\gamma$ phase. This condition seems to be the reason why the cuboidal $\gamma^{\prime}$ phase became unstable in Fig. 2(e).
Furthermore, the variation of the anisotropy affected the particle size distribution as shown in Fig. 4 and Table 1. Vaithyanathan and $\mathrm{Chen}^{2)}$ studied the effect of precipitate volume fraction on the particle size distribution and showed that the increase in volume fraction of the $\gamma^{\prime}$ phase shifts the peak radius to smaller normalized radius and leads to broadening of the distribution. In the particle size distributions obtained in this study, the peak radius is similar, i.e., $r / \bar{r}(t)=0.7 \sim 0.8$ (see Figs. 4 and 5), which is consistent with the position reported by Vaithyanathan and Chen, ${ }^{2)}$ when the volume fraction of the precipitate is about $55 \%$. It is interesting that the variation of the anisotropy affected only width of the distribution spectrum without changing the peak radius as shown in Table 1 . It should be emphasized that the difference of about $20 \%$ in elastic constants between the $\gamma$ and $\gamma^{\prime}$ phases affected both precipitate morphology and particle size distribution (see Figs. 2 and 4).

The effect of the difference in the shear modulus between the matrix and the precipitate has been also reported previously, ${ }^{7,8)}$ but it was examined based on the same elastic constants for both the matrix and the precipitate. In this study, however, the elastic constants for each phase are based on the practical ones for TMS-26, and the variation of the shear modulus did not affect both morphology and particle size distribution of the $\gamma^{\prime}$ phase. This is probably because the anisotropy in the two phases affected mainly the stability of the $\gamma^{\prime}$ morphology. In other words, under the conditions of the variation of the shear modulus employing in this study, the cuboidal $\gamma^{\prime}$ phase was stable by the effect of the anisotropy in the $\gamma$ and $\gamma^{\prime}$ phases. 
It is known that the elastic interactions affect the shape of the particle size distribution. ${ }^{2)}$ However, as long as we know, this is the first simulation showing that the elastic inhomogeneity affects the width of the size distribution spectrum.

\section{Conclusions}

2D phase-field simulation was carried out to examine the effect of elastic inhomogeneity on the microstructure evolution in Ni-based superalloys. Based on the elastic constants of a Ni-based superalloy, both anisotropy and shear modulus in the $\gamma$ and $\gamma^{\prime}$ phases were varied independently. The variation of the anisotropy affected the morphology of the $\gamma^{\prime}$ phase and caused the cuboidal shape unstable when the ratio of $\delta^{\mathrm{p}}$ to $\delta^{\mathrm{m}}$ was large. Furthermore, the variation of the anisotropy affected only width of the distribution spectrum of the $\gamma^{\prime}$ particle size without changing the peak radius. On the other hand, the variation of the shear modulus did not affect both morphology and particle size distribution of the $\gamma^{\prime}$ phase.

\section{REFERENCES}

1) Y. Wang, D. Banerjee, C. C. Su and A. G. Khachaturyan: Acta Mater. 46 (1998) 2983-3001.

2) V. Vaithyanathan and L. Q. Chen: Acta Mater. 50 (2002) 4061-4073.

3) J. Z. Zhu, T. Wang, S. H. Zhou, Z. K. Liu and L. Q. Chen: Acta Mater. 52 (2004) 833-840.

4) J. Z. Zhu, T. Wang, A. J. Ardell, S. H. Zhou, Z. K. Liu and L. Q. Chen: Acta Mater. 52 (2004) 2837-2845.

5) S. V. Prikhodko, J. D. Carnes, D. G. Isaak, H. Yang and A. J. Ardell: Metall. Mater. Tran. A 30 (1999) 2403-2408.

6) K. Tanaka, T. Kajikawa, T. Ichitsubo, M. Osawa, T. Yokokawa and H. Harada: Mater. Sci. Forum 475-479 (2005) 619-622.

7) P. H. Leo, J. S. Lowengrub and H. J. Jou: Acta Mater. 46 (1998) $2113-$ 2130.

8) S. Y. Hu and L. Q. Chen: Acta Mater. 49 (2001) 1879-1890.

9) S. G. Kim, W. T. Kim and T. Suzuki: Phys. Rev. E 60 (1999) 71867197.

10) J. W. Cahn and J. E. Hilliard: J. Chem. Phys. 28 (1958) 258-267.

11) A. G. Khachaturyan: Theory of structural transformations in solids, (Wiley, New York, 1983).

12) T. Mura: Micromechanics of Defects in Solids, 2nd revised ed., (Kluwer Academic, 1991).

13) I. Ansara, N. Dupin, H. L. Lukas and B. Sundman: J. Alloy. Compd. 247 (1997) 20-30.

14) A. J. Ardell: Acta Metall. 16 (1968) 511-516. 